\title{
Les systèmes de production agricole dans la chaîne agroalimentaire : position et évolution
}

\section{Sylvie Bonny}

\section{(2) OpenEdition}

\section{Journals}

\section{Édition électronique}

URL : http://journals.openedition.org/economierurale/2751

DOI : 10.4000/economierurale. 2751

ISSN : 2105-2581

\section{Éditeur}

Société Française d'Économie Rurale (SFER)

\section{Édition imprimée}

Date de publication : 2 août 2005

Pagination : $91-98$

ISSN : 0013-0559

\section{Référence électronique}

Sylvie Bonny, « Les systèmes de production agricole dans la chaîne agroalimentaire : position et évolution », Économie rurale [En ligne], 288 | Juillet-août 2005, mis en ligne le 05 juillet 2009, consulté le 19 avril 2019. URL : http://journals.openedition.org/economierurale/2751 ; DOI : 10.4000/ economierurale. 2751 


\title{
Les systèmes de production agricole dans la chaîne agroalimentaire : position et évolution
}

\author{
Sylvie BONNY • Institut national de la recherche agronomique, Grignon
}

De nombreuses voix soulignent aujourd'hui pour l'agriculture la nécessité de répondre aux attentes de la société, des consommateurs, des citoyens. Cette injonction largement reprise pose en fait diverses questions. En particulier, elle devrait mieux prendre en considération la position de l'agriculture dans la chaîne agroalimentaire et replacer les systèmes de production dans celle-ci, notamment vis-à-vis de l'aval et de la grande distribution. C'est cette idée que nous souhaitons développer dans cette Tribune.

Depuis quelques années ou décennies, diverses critiques sont formulées envers les systèmes de production agricole en France et en Europe. Sont ainsi souvent stigmatisés leur simplification, l'uniformisation génétique des races et variétés, l'agrandissement des ateliers animaux avec une forte concentration du bétail ou de la volaille, les pollutions, une détérioration de la qualité de l'alimentation, un appauvrissement des paysages, tout cela n'empêchant pas la nécessité de soutiens au revenu agricole. L'agriculture actuelle est parfois jugée « folle » et les systèmes de production peu durables, d'autant plus que de lourds capitaux sont nécessaires pour l'installation dans ce métier.

Dans le même temps, une très forte attente s'exprime envers l'agriculture en matière de nouvelles fonctions à remplir, de caractéristiques de l'alimentation, de préservation de l'environnement, de contribution à la lutte contre l'effet de serre, de satisfaction des demandes des consommateurs, d'entretien des espaces. La Recherche et le Développement sont, de leur côté, vivement incités à travailler sur cet objectif : quels systèmes de production proposer pour répondre à ces demandes, rectifier les défauts et relever les défis posés, en particulier pour s'orienter vers une agriculture plus durable?

Mais quels sont les facteurs qui déterminent les caractéristiques des systèmes de production ? La filière agroalimentaire est souvent perçue et analysée dans le simple sens de la circulation physique des biens, depuis les industries d'amont vers les consommateurs. Les critiques et les attentes exprimées semblent fréquemment renvoyer à une analyse découlant simplement de ce sens de la circulation des biens de l'agrofourniture vers les utilisateurs finaux. Pourtant les systèmes agricoles sont également et de façon croissante fortement déterminés par leur place dans la chaîne agroalimentaire, en particulier par rapport à l'aval et surtout par leur position dans la chaîne de valeur. Cette influence des acheteurs aval - industries de transformation et grande distribution, ensemble que l'on désignera globalement par le sigle TCD pour secteurs de la Transformation, Commercialisation et Distribution - mérite d'être mieux prise en considération. Cela peut permettre de mieux évaluer les marges de manœuvre, les possibilités de réponse des systèmes agricoles aux diverses demandes exprimées.

Nous développons cette proposition autour de trois points : l'importance des secteurs aval dans l'évolution des systèmes agricoles, la position économique de ces derniers dans la chaîne agroalimentaire et le rôle croissant de la grande distribution. 


\section{L'importance de la demande de l'aval dans l'évolution des systèmes agricoles}

\section{Orienter la production selon « la demande "...}

Les critiques adressées à l'agriculture en matière d'environnement ou de qualité des produits insistent souvent sur une nouvelle orientation nécessaire : la production agricole doit désormais être tournée vers la satisfaction des attentes des consommateurs et de la société et être pilotée par celles-ci. Autrement dit, selon la terminologie anglo-saxonne - citée ici car cette tendance est internationale -, l'agriculture doit passer d'une approche "supply push" à une approche "demand driven". Ce mot d'ordre et ce leitmotiv paraissent faire l'objet d'un consensus quasi général même si les formes d'agriculture envisagées pour cela varient selon les acteurs. Pourtant l'analyse de la notion de demande en économie standard est souvent relativement pauvre. Elle s'interroge assez peu sur ses processus d'élaboration et de construction, et surtout sur ses impacts. La demande est considérée comme un but qu'on doit seulement viser à satisfaire de façon à adapter l'offre au niveau de demande découlant des prix et de l'élasticité des quantités voulues quand ceux-ci varient. C'est déjà ce qu'exprimait Adam Smith il y a 230 ans dans la Richesse des Nations (1776): "Consumption is the sole end and purpose of all production; and the interest of the producer ought to be attended to, only so far as it may be necessary for promoting that of the consumer." Les principaux déterminants de la demande pris en compte relèvent des prix et des revenus, des différenciations des produits et de leur caractérisation selon la façon dont les consommateurs peuvent en évaluer la qualité par leur expérience, par recherche d'informations ou par confiance dans les attributs affichés. La diversité des demandes est surtout prise en considération par la segmentation des marchés. Par ailleurs diverses externalités de la consommation sont quasi ignorées, par exemple au niveau environnemental, alors que celles de la production font désormais l'objet d'investigations et d'actions.
Un mécanisme de fait déjà ancien

Cet objectif pour l'agriculture d'être guidée par la demande semble oublier ou sousestimer que dans une large mesure elle l'a bien été durant ces dernières décennies. La demande qui a alors joué un rôle est en particulier celle des IAA (industries agroalimentaires assurant la transformation des produits) et surtout de façon de plus en plus importante celle de la grande distribution. Les divers exemples du tableau 1 montrent ainsi que les systèmes de production mis en œuvre durant les dernières décennies ont bien été pilotés par «la demande », du moins celle de certains acteurs.

La majorité des agriculteurs ont cessé progressivement leurs activités de transformation à la ferme et de commercialisation directe et ont joué de façon croissante un simple rôle de fournisseurs/livreurs de matières premières de base à bas prix. De leur côté les secteurs aval des IAA, de la commercialisation, du marketing et de la distribution se sont considérablement développés. Si cette évolution est bien connue, ses retombées sont assez souvent mal prises en compte. La demande de l'aval a entraîné dans un premier temps une « commodification » des produits livrés par l'agriculture, i.e. leur passage à un statut de matières premières banales de base (commodities), ainsi que leur homogénéisation et leur standardisation. En outre, elle a amené une modification de certaines de leurs caractéristiques pour être mieux adaptées à la transformation par les IAA, aux transports, aux critères de conservation, etc. Par exemple, la diminution du nombre de races animales ou de variétés cultivées n'est pas due au seul choix des agriculteurs. Ultérieurement, la demande de l'aval s'est portée vers plus de différenciation dans la gamme de chaque produit suite au développement de stratégies de différenciation de leur offre vers les consommateurs. D'autres exigences de l'aval influant sur les systèmes de production sont synthétisées dans le tableau 1. 


\section{Sous l'effet de la pression de l'aval...}

\section{...Baisse des prix agricoles}

Un des impacts les plus notables de la pression de l'aval a été la baisse des prix agricoles - qui a permis en quelque sorte sa propre expansion - et cela d'autant plus que l'approvisionnement s'est fait de façon croissante à l'échelle mondiale et non plus locale, afin d'obtenir des matières premières agricoles à bas prix. Pour compenser la baisse de valeur et des cours, les agriculteurs ont dû accroître les volumes vendus, agrandir leurs exploitations et réduire les coûts par unité de produit livré. Cela a été un des moteurs essentiels de l'évolution et correspond à une réponse aux exigences des clients (IAA et grande distribution) cherchant des matières premières peu coûteuses. Mais cette croissance de la taille des activités quasi indispensable pour faire face à la baisse des prix des produits livrés a nécessité des investissements en bâtiments, en matériel, en connaissances, etc. Cela n'était guère possible en plusieurs domaines sur la même exploitation d'autant plus que l'exode rural réduisait les ressources en main-d'œuvre familiale. Aussi les systèmes de production se sont spécialisés et simplifiés. Les retombées en sont notables, notamment sur le plan écologique avec la dissociation des cultures et des élevages. Le phénomène de localisation des productions a été accentué par l'implantation des industries d'approvisionnement là où les intrants étaient aux prix les plus bas comme dans les zones portuaires (Bretagne) - et par l'emplacement des industries de transformation. D'où la spécialisation régionale qui a elle-même des impacts environnementaux forts, avec excès de bétail en certaines zones et quasi-absence ailleurs.

Bien des producteurs agricoles réagissent à la baisse des prix de leurs produits en accroissant les volumes livrés, aussi l'offre est-elle souvent forte. Ce niveau d'offre à son tour renforce la tendance à la chute des cours. D'où une spirale de production souvent élevée entraînant de bas prix, et ainsi de suite. Cela a induit l'apparition de surplus agricoles, aussi a-t on souvent dénoncé la mauvaise adaptation des volumes produits à la demande des marchés. Outre les aléas climatiques, ce phénomène a pu être renforcé par certaines politiques, mais il découle aussi d'une mobilité plus difficile quand on est installé en agriculture. En effet une fois les investissements engagés, des utilisations alternatives des ressources (capital, travail, terre, équipements, savoirs) ne sont pas toujours accessibles, ce qui fait qu'une réponse logique en théorie, la mobilité vers d'autres secteurs, - bien qu'elle soit forte au changement de génération ou à long terme - ne l'est pas de façon suffisante pour freiner hausse de la production et baisse des prix. À plus long terme les producteurs qui se maintiennent ou ceux qui s'installent, doivent avoir des coûts de production bas. Aussi l'augmentation de la taille moyenne des ateliers souvent critiquée est de fait difficile à enrayer, sauf quand la transformation et/ou la vente directe des produits sont possibles. Ainsi les bas prix des produits agricoles sont une cause notable de deux phénomènes couramment décriés mais dont les déterminants sont souvent assez mal analysés, le «productivisme » et aussi de la nécessité de soutiens au revenu.

\section{et diverses externalités négatives}

Depuis quelques années ou décennies il y a eu de fréquentes dénonciations des externalités négatives des systèmes agricoles, notamment en matière d'environnement, de qualité, de conditions d'élevage, de taille, etc. Explicitement ou implicitement on en fait très souvent porter les responsabilités (a) sur les choix des agriculteurs eux-mêmes, (b) sur la pression de l'amont et de l'agrofourniture, en particulier de l'agrochimie souvent mise à l'index, (c) sur les progrès techniques, et par là sur les orientations de la Recherche et du Développement dans les décennies après la Seconde Guerre mondiale. On méconnaît ainsi que l'évolution des systèmes, leurs orientations et leurs choix techniques ont été aussi fortement déterminés par la pression de l'aval, en particulier en matière de prix, d'homogénéité, de composition, etc. ( $c f$. tableau 1). 
Tableau 1. Exemples d'évolution des systèmes de production liée à la demande des IAA et de la grande distribution durant les dernières décennies

\begin{tabular}{|c|c|}
\hline $\begin{array}{l}\text { Facteurs de pilotage par la demande des IAA } \\
\text { et de la grande distribution (GD) }\end{array}$ & $\begin{array}{l}\text { Influences sur l'évolution } \\
\text { des systèmes agricoles }\end{array}$ \\
\hline $\begin{array}{l}\text { - Forte croissance des IAA et de la GD traitant } \\
\text { une proportion croissante des produits agri- } \\
\text { coles. Elles captent une part grandissante de la } \\
\text { valeur ajoutée liée à la transformation et à la } \\
\text { commercialisation, les prix des matières pre- } \\
\text { mières agricoles baissant de leur côté. }\end{array}$ & $\begin{array}{l}\text { - Production de matières premières } \\
\text { brutes avec chute de la transforma- } \\
\text { tion à la ferme et de la vente directe. }\end{array}$ \\
\hline $\begin{array}{l}\text { - Les IAA et la GD recherchent des matières } \\
\text { premières agricoles peu coûteuses. Comme l'ap- } \\
\text { provisionnement se fait sur le marché mondial } \\
\text { avec de bas prix de transport, la concurrence au } \\
\text { niveau des produits agricoles de base est vive. } \\
\text { D'où forte pression à la baisse des coûts. }\end{array}$ & $\begin{array}{l}\text { - Pour réduire les coûts unitaires de } \\
\text { production, on cherche à produire } \\
\text { plus par travailleur. D'où emploi de } \\
\text { races et variétés plus productives, } \\
\text { hausse de la taille des exploitations et } \\
\text { ateliers, etc. }\end{array}$ \\
\hline $\begin{array}{l}\text { - Les IAA demandent des produits adaptés à la } \\
\text { transformation et la GD s'approvisionne en } \\
\text { variétés et produits à bonne capacité de conser- } \\
\text { vation et ne retient que ceux ayant cette carac- } \\
\text { téristique. }\end{array}$ & $\begin{array}{l}\text { - choix des variétés adaptées à ces } \\
\text { demandes, d'où modification de cer- } \\
\text { taines caractéristiques de qualité des } \\
\text { produits. }\end{array}$ \\
\hline $\begin{array}{l}\text { - Les IAA et la GD recherchent des matières } \\
\text { premières agricoles assez standardisées pour } \\
\text { faciliter la transformation (grâce à une matière } \\
\text { première homogène) et la commercialisation } \\
\text { (calibrage, normes de classement des produits } \\
\text { pour les échanges, etc.). Elles sélectionnent } \\
\text { indirectement les variétés adaptées à ces objec- } \\
\text { tifs. }\end{array}$ & $\begin{array}{l}\text { - Les variétés végétales et les races } \\
\text { animales s'uniformisent et se rédui- } \\
\text { sent en nombre dans une première } \\
\text { phase. }\end{array}$ \\
\hline $\begin{array}{l}\text { - Le secteur aval développe ces dernières années } \\
\text { une stratégie de différentiation de son offre aux } \\
\text { consommateurs et accroît pour cela la contrac- } \\
\text { tualisation avec les producteurs. }\end{array}$ & $\begin{array}{l}\text { - Plus récemment adaptation plus } \\
\text { fine à diverses demandes selon des } \\
\text { critères de qualité spécifiés par l'aval. }\end{array}$ \\
\hline $\begin{array}{l}\text { - Tout fruit ou légume très légèrement taché ou } \\
\text { abîmé serait refusé, de même que diverses pro- } \\
\text { ductions ayant un certain taux de mycotoxines. } \\
\text { Le paiement selon divers critères de qualité } \\
\text { (composition) se généralise. }\end{array}$ & $\begin{array}{l}\text { - Les traitements phytosanitaires } \\
\text { sont assez nombreux pour pouvoir } \\
\text { atteindre ces normes, les apports azo- } \\
\text { tés visent aussi à obtenir un volume } \\
\text { suffisant avec la composition requise. }\end{array}$ \\
\hline
\end{tabular}




\section{La position économique des systèmes agricoles dans la chaîne agroalimentaire}

Les agriculteurs, devenus simples fournisseurs de matières premières à bas prix, ont vu leur part régresser dans la chaîne agroalimentaire tandis que se développaient les secteurs aval de la transformation, de la commercialisation, du marketing et de la distribution. En corollaire la composition de l'alimentation a fortement changé : elle est de plus en plus transformée et la part des produits tout préparés, prêts à consommer ou à cuire a beaucoup augmenté, ainsi que la consommation hors foyer.

\section{Prix agricoles et prix alimentaires}

...valeur de la production agricole et dépenses alimentaires...

Si les prix des produits agricoles ont chuté à monnaie constante, ceux des denrées alimentaires ont légèrement progressé ou sont demeurés quasi stables hors inflation (figure 1). L'évaluation des prix alimentaires est certes approchée car elle intègre à la fois le trend des prix des produits et la modification de la composition du «panier de la ménagère »; mais les deux phénomènes sont difficiles à dissocier. Si l'on répartit le montant total des dépenses alimentaires des consommateurs en deux grands types de postes (1) la valeur des produits agricoles vendus par les exploitants, (2) l'ensemble des dépenses liées à la transformation et commercialisation des produits en incluant transport, emballage, frais de marketing et de publicité, on observe une baisse de la part des premiers dans la consommation finale des ménages (figure 2). Le pourcentage des dépenses alimentaires revenant au secteur agricole aux États-Unis n'a cessé de se réduire depuis plusieurs décennies (USDA-ERS, 2002) $)^{1}$. Il en est de même en France : la

1. USDA-ERS. Briefing room: food marketing and price spreads. USDA-ERS (US Dept of Agriculture, Economic Research Service), 2002 www.ers. usda.gov/Briefing/FoodPriceSpreads/trends/ part des produits agricoles dans les dépenses alimentaires globales des ménages ne représente plus aujourd'hui qu'une proportion très faible du fait des changements de composition de l'alimentation et de la chute des prix agricoles à monnaie constante.

Figure 1. Évolution des prix agricoles et des prix alimentaires depuis 40 ans

(indice 100 en 1970, monnaie constante)

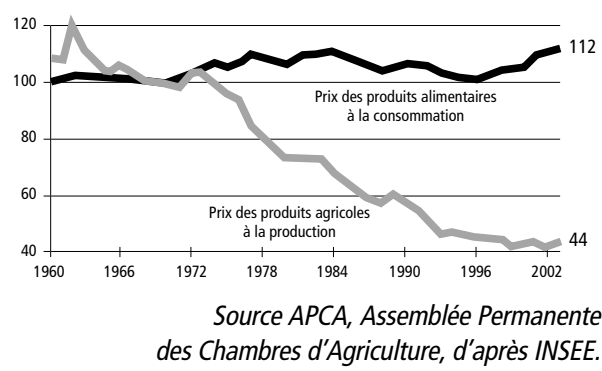

Figure 2. Répartition des dépenses alimentaires entre produits agricoles et activités de transformation/ commercialisation/distribution depuis $\mathbf{5 0}$ ans aux États-Unis (en milliards de dollars constants)

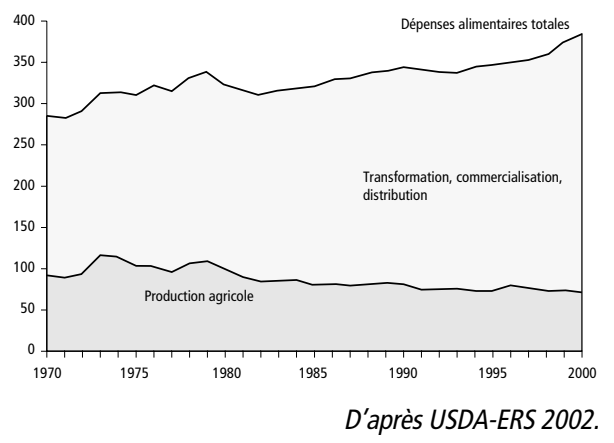

\section{Répartition des dépenses alimentaires}

...une expansion de la part de l'aval...

Globalement, en dollars constants, les dépenses alimentaires totales ont augmenté de $35 \%$ environ entre 1970 et 2000 aux USA. Si on les calcule par habitant depuis 1950, elles ont aussi progressé aux USA comme en France. Mais dans le budget total des ménages, la part de l'alimentation a chuté en moyenne sur la même période : ainsi le poste « alimentation, tabac, alcools » 
représentait $44 \%$ des achats des ménages en 1949, $38 \%$ en 1959 mais moins de $18 \%$ en 2003 en France. Cette réduction est due à la progression d'autres types de dépenses, non à une baisse des achats alimentaires en monnaie constante.

L'expansion du secteur TCD est aussi à rapprocher de l'évolution générale des modes de vie : proportion croissante de femmes travaillant à l'extérieur, recherche de repas vite préparés pour les jours ordinaires, etc. Ainsi David Hughes, professeur de marketing alimentaire à l'Imperial College de Londres estimait que le temps moyen quotidien consacré à la préparation des repas à domicile au Royaume-Uni était passé de 2 h 30 dans les années 1930 à 15 mn vers le milieu des années 1990 (Grievink, 2003) ${ }^{2}$. L'évaluation faite aux USA dans les enquêtes d'emploi du temps est plus élevée, mais témoigne aussi d'une baisse sensible : en moyenne $44 \mathrm{mn}$ en 1965, 27 mn en 1995 pour les personnes de 18 à 64 ans. En d'autres termes, les emplois dans l'agroalimentaire et la distribution ont remplacé le travail paysan de transformation - commercialisation et le travail domestique de préparation des repas.

\section{...mais avec des effets sur l'environnement et la santé}

Il est certes connu que nombre d'activités de transformation/préparation alimentaires faites naguère à la ferme ou à la maison sont désormais assurés par le secteur TCD. Mais les multiples retombées de cette évolution sont souvent peu prises en compte. En matière d'impacts sur l'environnement et la santé, jusqu'à une période récente le secteur aval a fait l'objet d'assez peu de questionnements, notamment par les médias et le grand public, comparativement à ceux envers l'agriculture et son amont. Pourtant

2. Grievink J.-W. The changing face of the global food supply chain. Conférence de l'OCDE “Changing Dimensions of the Food Economy: Exploring the Policy Issues", La Haye, 6-7 février 2003. webdomino1.oecd.org/comnet/agr/foodeco.nsf il peut avoir des effets considérables en la matière. Ainsi les transports qui se sont multipliés à tous les stades de la filière avec la recherche de matières premières à bas prix partout dans le monde et l'internationalisation de la distribution, ont de lourdes répercussions énergétiques et environnementales, de même que la multiplication des emballages et des supports publicitaires. Certes, si la région d'origine d'un produit dispose d'un net avantage comparatif agroécologique, le coût énergétique et environnemental du transport peut être contrebalancé par un moindre coût à la production en ce domaine, mais cela est loin d'être toujours le cas.

\section{Qu'en est-il de la qualité des produits alimentaires transformés ?}

En la matière, l'adjonction de constituants moins coûteux (e.g. sel, sucres, matières grasses, épaississants, édulcorants, agents de texture, de sapidité, de moelleux, etc.) qui, à poids égal se substituent en partie aux matières premières originelles, a une influence notable sur les caractéristiques de composition, de goût et surtout d'équilibre nutritionnel. Le secteur aval propose ainsi assez souvent une alimentation plutôt déséquilibrée au niveau diététique. En effet les denrées transformées commercialisées contiennent fréquemment un excès de sel, de sucres rapides et de lipides qui en améliorent la sapidité tout en en diminuant le coût. Mais cela favorise diverses pathologies alimentaires liées à une consommation excessive de ces composants, ainsi que la hausse de la prévalence de l'obésité que l'on observe actuellement. Les consommateurs ont certes une grande latitude de choix au niveau alimentaire et donc une part de responsabilité individuelle. Mais la question de la composition relativement déséquilibrée au niveau diététique des denrées transformées ne peut pas être éludée, d'autant plus que les habitudes alimentaires s'acquièrent très jeunes et que les impacts sur la santé de produits alimentaires transformés trop riches 
en sel, sucres et graisses cachées sont considérables.

Une autre influence notable de la TCD est la pression qu'elles exercent d'une part pour s'approvisionner à bas prix, d'autre part pour conquérir des parts de marché quasiment partout dans le monde et dans tous les secteurs. Ainsi la grande distribution s'implante dans les pays en voie d'industrialisation et en développement (Reardon et al., 2003) ${ }^{3}$, de même que la restauration rapide. Si l'installation de supermarchés dans les villes des pays émergents peut avoir des impacts positifs pour certains consommateurs, elle a également un effet déstructurant sur les systèmes de production et transformation agricoles locaux, mais aussi sur les modèles alimentaires. Dans certains pays émergents, du fait de l'industrialisation rapide de l'alimentation, les maladies chroniques liées (problèmes de surpoids, d'obésité, de diabète, d'atteintes cardio-vasculaires) atteignent déjà des niveaux inquiétants pour une part croissante de la population (Maire, Delpeuch, 2004) ${ }^{4}$.

\section{Pouvoir et dynamiques d'évolution dans la chaîne agroalimentaire}

La chaîne agroalimentaire s'est complexifiée et allongée et a une structure fort hétérogène : elle est composée d'acteurs dont la dimension économique et le nombre à chaque niveau sont très disparates. Grievink $^{5}$ en a établi une représentation en sablier qui l'illustre de façon éclairante dans le cas de l'Europe (figure 3).

3. Reardon T., Timmer C.-P., Barrett C.-B., Berdegué J. The Rise of Supermarkets in Africa, Asia, and Latin America. American Journal of Agricultural Economics, 2003, 85 (5), p. 1140-1146.

4. Maire B., Delpeuch F. La transition nutritionnelle, l'alimentation et les villes dans les pays en développement. Cahiers Agricultures, 2004, 13 (1), p. 23-30. 5. Op. cit. Voir note 2.
$\mathrm{Au}$ sommet se trouvent les consommateurs très nombreux mais hétérogènes. Près de la base et de l'autre extrémité, les agriculteurs sont atomisés et de dimension économique généralement modeste. Le groupe d'acteurs central, à la hauteur du goulot d'étranglement, a une taille et un pouvoir économique sans commune mesure du fait de son faible nombre : il s'agit des grandes centrales d'achat des groupes de la distribution.

Leur pouvoir est notable car une part croissante des acteurs et firmes situés à leur amont doivent passer par leur intermédiaire pour pouvoir mettre leurs produits sur le marché. Le secteur de la grande distribution s'étant beaucoup concentré, quelques grandes centrales de distribution deviennent un point de passage de plus en plus obligé pour pouvoir commercialiser les produits. Or souvent, du fait de la vive concurrence entre elles, elles ont des stratégies agressives en termes d'expansion, d'approvisionnement auprès de leurs fournisseurs et de nouvelles implantations dans des pays émergents. Elles imposent aussi à leurs fournisseurs des conditions, par exemple de plus en plus des normes et exigences techniques à satisfaire (les « private standards ») pouvant entraîner l'exclusion d'une partie d'entre eux. Cela peut concerner des IAA devant verser des « marges arrières » pour que leurs marchandises soient référencées dans les grandes surfaces, et aussi des producteurs ou groupements de producteurs pour satisfaire à des normes techniques. Ceci touche aussi les producteurs du Sud comme les grands groupes de la distribution s'y développent.

La grande distribution a également une forte influence sur les consommateurs, le milieu naturel et, plus généralement, les modèles de développement socio-économique qui s'orientent vers la progression des consommations achetées et la «marchandisation »d'un nombre croissant de biens. 


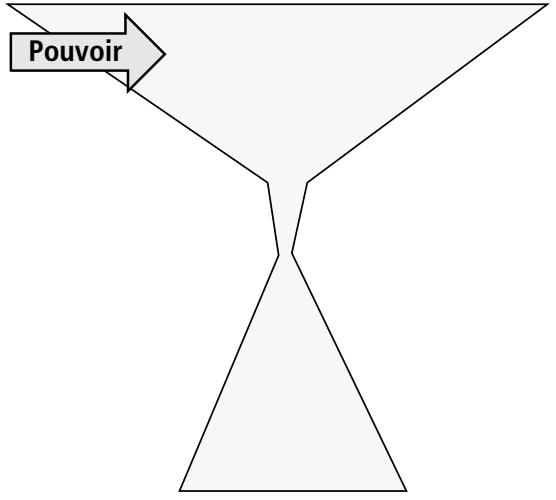

Consommateurs :

Acheteurs :

Points de vente :

Supermarchés :

Centrales d'achat :

Industries alimentaires :

Industries de première transformation : 80000

Négociants :

Agriculteurs :
160000

160000000

89000000

170000

600

8600

3200000

Source : Grievink, 2003

\section{Que faut-il conclure ?}

Il semble important de mieux prendre en compte la part respective du secteur agricole et du secteur aval, et l'influence croissante de ce dernier dans l'analyse de diverses questions. Ainsi les interrogations sur la durabilité environnementale, la qualité de l'alimentation, les impacts sur les pays du Sud semblent parfois trop exclusivement adressées à l'agriculture, et insuffisamment à l'ensemble de la chaîne. Plus exactement elles sont souvent adressées à l'agrofourniture (pour l'emploi des intrants) ou au secteur agricole lui-même (à propos de l'agrandissement, de l'environnement, de la taille des ateliers, du bien-être animal, etc.). Cela méconnaît la dépendance de l'agriculture envers son aval, et notamment de plus en plus le rôle de la grande distribution, qui impose prix, normes, spécifications techniques, volumes... Cela semble aussi ignorer la part de l'aval en matière de pollution et de qualité nutritionnelle des denrées.

Ainsi les critiques fréquentes exprimées par les associations environnementalistes ou par les médias à l'agriculture actuelle semblent parfois assez myopes car, focalisées sur celle-ci et son amont, elles méconnaissent souvent les pressions de l'aval influant sur les orientations agricoles. Certes la grande distribution met constamment en avant son souci de répondre aux désirs des consommateurs par les prix, la commodité d'emploi et diverses autres caractéristiques des produits proposés. Mais les contreparties sur l'amont et l'agriculture, et les répercussions sur l'environnement ou la santé, ne doivent pas être ignorées.

Bien des menaces pèsent sur l'avenir de la planète, entre autres au niveau environnemental. L'agriculture occupe une position écologique remarquable : captation grâce la photosynthèse de gaz carbonique et production de molécules organiques simples ou complexes, mise en valeur et entretien de l'espace, production alimentaire et production de ressources non alimentaires renouvelables. Elle pourrait de ce fait jouer un rôle important pour contribuer à une plus grande durabilité des activités humaines et de leurs impacts. Mais encore faut-il qu'elle en ait les moyens, et notamment que la chaîne agroalimentaire valorise mieux la production agricole 